\title{
Political, religious and occupational identities in context: Placing identity status
} paradigm in context

Olga Solomontos-Kountouri ${ }^{1}$, Jane Hurry ${ }^{2}$

School of Psychology and Human Development, Institute of Education, University of London, 25 Woburn Square, London WC1H 0AA, UK

\begin{abstract}
This study critically contrasts global identity with domain-specific identities (political, religious and occupational) and considers context and gender as integral parts of identity. In a cross-sectional survey, 1038 Greek Cypriot adolescents (449 boys and 589 girls, mean age 16.8) from the three different types of secondary schools (state, state technical and private) and from different SES completed part of the Extended Objective Measure of Ego-Identity Status-2 (EOMEIS-2). The macrocontext of Greek Cypriot society is used to understand the role of context in adolescents' identities. Results showed that Greek Cypriot young people were not in the same statuses across their global, political, religious and occupational identities. This heterogeneity in the status of global identity and of each identity domain is partially explained by differences in gender, type of school and SES (Socio-Economic Status). The fact that identity status is found to be reactive to context suggests that developmental stage models of identity status should place greater emphasis on context.
\end{abstract}

\footnotetext{
${ }^{1}$ Corresponding author: Research Associate (PhD) School of Psychology and Human Development, Institute of Education, University of London, 25 Woburn Square, London WC1H 0AA, UK. Present address: 28 Kouriou, Dhali 2540, Nicosia, Cyprus. Tel.: 0035722524202.

E-mail address: olga s_k@hotmail.com

${ }^{2}$ Senior Lecturer, School of Psychology and Human Development, Institute of Education, University of London, 25 Woburn Square, London WC1H 0AA, UK. Tel.: 00442076126931; fax: 00442076126304

E-mail address: j.hurry@ioe.ac.uk
} 


\section{Introduction}

Contemporary Greek Cypriot society creates an interesting context for examining adolescents' identity, as it is a meeting point of tradition and modernization. Firstly, the national issue of a partitioned country places pressures on young people to deal with politics since it is always on the politicians' agenda, it is regularly presented by the mass media and it is implicated in education. Secondly, Christian Orthodox religion is a strong institution, which is influencing most aspects of Greek Cypriots' lives, although it is in contrast with their modernised life style. Thirdly, the affluent economy of Cyprus (especially the tourist industry and the consequences of joining the European Union) creates potentials for young people. In such a context the domains of politics, religion and occupation are more salient to Greek Cypriot young people, in contrast to their western counterparts. This study adopts Erikson's essential position that ego identity develops in close relation to the adolescent's relevant socio-historical context and focuses not only on the macrocontext (politics, religion, economy) but also on meso-contextual factors (academic and economic capital) and even micro-contextual factors (gender). It also advocates the use of the domain specific identities as they are more accurate about what they represent (Meeus, 1996).

Placing Identity Status Paradigm in context

Marcia (1966), based on Erikson's theory, developed the Identity Status Paradigm (ISP) that dominates identity research. According to Erikson (1968) adolescents are experimenting with alternative roles and ideals available in their 
society before making relatively enduring commitments, which provide them with a secure sense of identity within their community. Marcia (1966) following Erikson, viewed identity as a global construct and he initially aggregated it from the domains of occupation, politics and religion. Using a semi-structured interview he measured four identity statuses as follows: Adolescents who have gone through a period of exploration and have established firm identity commitments are assigned to Achievement status. Adolescents who are actively seeking among alternatives to arrive at a choice are in Moratorium status. Adolescents who follow the commitments that are presented to them by parents (or other significant adults), without exploring options, are in Foreclosure status. Finally, identity Diffusion status refers to adolescents who have no commitments and who are not actively trying to form any (Marcia, 1980).

Contextual influences on identity statuses had been largely neglected in empirical research. As Cote and Levine (1988) identified, the ISP ignored the role of the context and viewed statuses as an intrapersonal attribute, whose development is mainly affected by individual factors. They further suggested that Marcia's notion of identity commitment is rather a-contextual while Erikson's social psychological view of commitment formation is context-related. For Erikson, the adolescent's commitment to (his/her) identity is not complete unless "society... recognising him as somebody who had to become the way he is" (Erikson, 1980: 122).

Nevertheless, there are some serious attempts by ISP theorists to put individuals' identity in context. Yoder (2000) introduced the concept of identity "barriers;" context and time specific limitations such as Socio-Economic Status, educational opportunities, political restrictions, religion and gender, which are imposed externally and limit the individual's exploration or/and commitment in all or 
some domains. She suggested that ISP researchers should empirically identify these barriers in each study and separate them from internal limitations. Adams and Marshall (1996) proposed that interpersonal communications, culture, economics, politics and social class all influence identity formation through shared values and ideologies. Despite this, in empirical investigation, there is a focus on the microcontextual influences on identity formation, such as the contribution of "significant others" (e.g., Kerpelman, Pittman, \& Lamke, 1997). However, a deeper understanding of the contextual influences on identity formation should be achieved by studying also the macro- and meso-contextual influences.

\section{Macro-contextual influence on identity}

Baumeister (1986) theorised how different societies or the same society at different times, provide different levels of choice in the construction of identity. Similarly, Cote and Levine (2002) encountered the equivalence of identity statuses within different types of society. In pre-modern societies individuals are expected to be in Foreclosure status by accepting the expectations of other people. In modern societies, individuals are expected to be in Achievement status by following their distinct personal style. In late-modern societies, Diffusion and Moratorium statuses emerge as individuals project images that meet the approval of a community as long as the images remain acceptable. Few ISP studies have drawn attention to understanding identity within the specific macro-context in which a study takes place. For example, investigating the impact of social and economic changes (Kroger, 1993) and the impact of specific societies (Jensen, Kristiansen, Sandbekk, \& Kroger, 1998; Stegarud, Solheim, Karlsen, \& Kroger, 1999). Also, the bulk of identity studies have 
been conducted using US samples, limiting our understanding to identity formation within this "dominant" culture. Schwartz (2005) calls researchers to study identity in other cultural contexts to advance our knowledge of the interaction between specific cultural processes and identity advancements.

The particular historical, social and cultural context of Greek Cypriot society provides an interesting macro-context within which to examine identity formation and to compare to adolescent identity in Western cultures. More specific information on the tense political situation, the strong value base of religion and the affluent economy are presented to introduce to the context of the study.

A combination of forces constructs a highly politicised environment for young people, especially young men. Historically, Greek culture introduced the concept of democracy. The civic responsibility to actively participate in the democratic process is still a feature of Greek Cypriot society, where, for example voting is compulsory. In addition, the "Cyprus Problem" embedded deep in Cypriot history, involving the confrontation of Greek and Turkish Cypriot inhabitants of the island, creates a tense and highly visible political climate. Moreover, the Greek Cypriot political parties are quite polarised, putting extra pressure, especially on young males who are expected to be politically engaged.

The Christian Orthodox Church of Cyprus is a powerful institution that exerts influence on people's spiritual, cultural and social lives. Most of traditional customs are related to religious celebrations round the year; church weddings, baby christenings, Sunday services and participation in the sacramental life of the church are the foundations of Christian Orthodox families (Alevisopoulos, 1994). In these families, bringing up children and adolescents within church tradition is considered 
one of the basic parental duties. Women are culturally considered as more religious than men and are also mostly responsible for keeping religious traditions.

In the domain of occupation there are also unique contextual influences.

During the last two decades, the free enterprise economical system, has transformed Cyprus from already traditional community to the16th place worldwide in terms of per capita income (Cyprus, 2002). These developments are creating high occupational aspirations amongst adolescents. However, the requirement that young men serve the army for 26 months after leaving school creates a gap between them and their female contemporaries in terms of selecting future careers.

\section{Meso-contextual influence on identity}

Within a particular macro-context, different meso-contextual variables exert their own effect on identity formation. Type of education has been acclaimed as key meso-contextual variable (Cote \& Levine, 1988). Levine (see Levine, Pakvis, \& Higgins-D'Alessandro, 2000) conducted a few studies to examine the impact of technological and the humanistic university orientation on identity formation. They

found that: technological oriented students are likely to experience less severe identity crises, in contrast to humanistic oriented students. Levine et al (2000) explained that the technological university orientation provides more support to the students to simply define themselves in terms of their occupations; this orientation is also encouraged by North American society, while humanistic university orientation provides a more marginal ideology often opposed by the broader social system. Adams and Fitch (1983) showed that educational environments that promoted thought expanding, analytical and critical awareness of societal issues facilitated 
identity formation. They challenged the extent of the impact of educational environments on university students' identity by showing that different university departments attracted students with different identity status. Therefore, there is a need for studying secondary education students, where there is a broader spectrum of academic abilities and a non-biased range of identity statuses. There are also other meso-contextual factors that have to be investigated. In particular, Phillips and Pittman (2003) call identity researchers to examine the relationship between identity and Socio-Economic Status (SES), which has been ignored. On a theoretical article based on literature on poverty and identity, they proposed that poverty in children and adolescents that is associated with stress, social stigma, and limitations in opportunity should affect both identity exploration and identity commitment.

\section{Gender and identity}

Although gender is an integral part of identity, the general agreement from ISP empirical evidence on gender points to no significant differences in how and when females and males form their adult identities (Marcia, 1993; Meeus, Iedema, Helsen, \& Vollebergh, 1999; Waterman, 1993a). In a major review of 56 empirical studies (from 1966-1995) using various ISP measures, Kroger (1997) found little evidence of gender differences on identity status distributions between males and females. Both genders showed increasing frequencies of Moratorium and Achievement statuses and decreasing frequencies of Diffusion and Foreclosure statuses over time, although, half of the studies involving high school samples found that males undertook the above pathway later. These findings are consistent with the universalism of ISP, which assumes the same developmental process amongst individuals. 
However, gender differences are uncovered with the use of identity domains. Though these patterns have not been consistent across studies, they provide some insight into the relationship between gender and identity formation. Archer (1993) reported that in the domains of sexuality, family/career prioritising and friendship, females were more likely to be in Moratorium and Achievement statuses, while males were more likely to be in Foreclosure and Diffusion statuses. Goossens (2001) showed that in occupation, females were more likely to be in Achievement status and males in Foreclosure status; and in politics, males were more likely to be in Achievement status and female in Diffusion status.

\section{Domain specific identities}

The utilization of domain specific identities gains much support from identity researchers. Many adherents of ISP have advocated the use of both global and domain specific identities (e.g., Archer, 1993; Jackson, Dunham, \& Kidwell, 1990; Kroger, 1993; Waterman, 1993b). A few ISP researchers propose the exclusive use of domain specific identities finding that global identity does not provide a representative picture of domain specific identities, (i.e., DeHaan \& Schulenberg, 1997; Goossens, 2001). Yet others have even treated identities as if they are independent constructs with growing bodies of literature and research: for example, ethnic identity (Phinney, 1989); occupational identity (Vondracek, Lerner, \& Schulenberg, 1983). Similarly, self-concept theory and research have a long tradition of utilising separately the domain specific self-concepts that are considered as distinguishable entities (Eccles, Adler, \& Meece, 1984). 
Although the examination of global identity, proposed by Erikson and Marcia, has been promoted as important in understanding the healthy development of young people, the measurement of global identity aggregated from different domains, has resulted in a number of empirical problems such as:

(a) Different criteria are used to weight domains specific statuses in calculating a global score. Marcia (1966) was unclear about the precise relationship between domain and global scores, leaving this to the judgement of the interviewer. Waterman (1993b) gave five different guidelines on how to obtain the global identity score (e.g., one criterion weights global identity to the domain that is important to the interviewee; another criterion weights global identity to the intuitive impression of the interviewer). In the EOMEIS, an additive integration of the domain-specific ratings is used.

(b) Statistical tests of the convergence of identity statuses across domains and between global and domain specific statuses do not support that global identity status represents each identity domain. First, the convergence across domains is very low. For example, in Pastorino et al's (1997) identity-interview study, in which six domains were covered, only $2.4 \%$ of a college sample was classified in the same status group across domains. Second, the convergence between global and domainspecific statuses is moderate: $69.4 \%$ for politics (Kroger, 1986), 58.8\% for occupation and higher (85\%) for religion (Rogow, Marcia, \& Slugoski, 1983).

(c) The inclusion of different identity domains in different studies are rather arbitrary and make comparisons difficult. For example, Marcia’s (1966) original interview includes the domains of occupation, politics and religion. Grotevant and Cooper's (1981, cited in Grotevant, Thorbecke, \& Meyer, 1982) expansion of Marcia's interview includes three more domains: friendship, dating and sex roles 
domains. Grotevant and Adams (1984) in EOMEIS (Extended Objective Measure of Ego Identity Status) include eight domains and separate them into the ideological and the interpersonal (see also models of Balistreri, Busch-Rossnagel, \& Geisinger, 1995; Meeus, 1996). Identity domains are usually imposed by the researchers with the preassumption that ISP domains are relevant to all adolescents of any national background. However, self-concept theorists have shown that certain sub-domains of self-concept are more important to individuals' overall sense of self than others (Harter, 1996; Stryker, 1987). From this perspective, it is essential for identity studies to establish which identity domains are salient in the chosen context.

\section{Hypotheses of the present study}

The primary aim of this study is to advance our understanding of how the different levels of context of a non-examined culture influence identity formation. Secondly, it examines whether domain specific identities provide more valid and reliable information than global identity in a specific cultural context, where the domains of politics, religion and occupation are important for the particular adolescents. The study overcomes two limitations of other identity studies; the overuse of university students from Western countries (Cote, 1996; Schwartz, 2005) and the unexplored variable of Socio-Economic Status of participants (Phillips \& Pittman, 2003). Based on our reading of the literature and our knowledge of the Greek Cypriot context we hypothesise that:

1. Global identity status would not represent domain specific identity status of occupation, religion and politics of Greek Cypriot adolescents. 
2. Young Greek Cypriots would explore or/and form commitments in the domains of politics and religion to a greater extent than their North American counterparts.

3. Young females would be in more "advanced" identity statuses in the domains of occupation and religion than young males;

4. Young males would be in more "advanced" status in the domain of politics than young females.

5. Students from state technical schools would be more "advanced" in their occupational identity than students from state and private schools.

Whilst we consider SES to be an important meso-level variable, therefore worthy of exploration, there is insufficient information to generate any reliable hypothesis.

\section{Method}

Design and sample

The study utilised a cross sectional survey with a highly representative sample of students in the last year of secondary school in 2000-2001. The great majority of students in Cyprus stay until the end of secondary school.

A multi-stage cluster with the incorporation of proportionate stratification sampling was employed to draw a 10\% sample of the population (Moser \& Kalton, 1977). On the first level, stratified samples from the three different types of Greek Cypriot secondary education (state, state technical and private) were taken. On the second level, representation of all the geographic settings of Greek Cypriot adolescents was secured by taking stratified samples from five different provinces of Cyprus. On the 
third level, stratified samples from the different school specialisations (e.g., classical, scientific, economic, etc.) were taken, in order to secure representation of all students' educational background within each type of school. Participants were 1038 Greek Cypriot adolescents (449 males and 589 females) from 15 secondary schools. Their age ranged from 16 to 19 years with a mean age of 16.8. Socio-Economic Status (SES) was determined by both parents' occupations (Nash, 1995). Estimation showed that $22 \%$ of participants belong to upper class, $37 \%$ belong to middle class and $41 \%$ belong to working class. Thirty-two participants (3\%) withdrew from the study, as participation was voluntary.

\section{Procedure}

After the appropriate approvals from the Cyprus Ministry of Education and Culture, the first author (OSK) visited all the 15 schools by arrangement with the head teachers who helped with the third level of stratification (to get proportionate stratified samples from each school specialisation). She visited 49 different classes to introduce the research and administer the questionnaire. In general students were enthusiastic participants.

\section{Measure}

Information was collected on age, gender, name of the school, specialisation, geographical location, father's occupation and mother's occupation. 
Identity status was determined by means of a Greek adaptation (SolomontosKountouri, 2006) of part of the Extended Objective Measure of Ego Identity Status 2 (EOMEIS-2 Bennion \& Adams, 1986). EOMEIS-2 is the most current version of a self-report evolved from research experience with Marcia's (1966) semi-structured interview to allow for wider and more comparable measurement of the four identity statuses (Achievement, Moratorium, Foreclosure and Diffusion). Although EOMEIS2 is mainly used to measure global identity, the measurement of identity domains is also possible; and offers the opportunity to challenge the synchronicity of identity process in global and in domain specific identities by using the same measure. EOMEIS-2 was chosen as the most appropriate instrument for the purpose of the present study after piloting several identity measures.

EOMEIS- 2 consists of 64 items ( 8 items for each of 8 identity domains). For each domain there are two items per identity status. The participant is asked to indicate agreement or disagreement on a 6-point-likert scale, with the anchors of "strongly disagree" to "strongly agree." The present study utilised the 24 items that refer to the domains of politics, religion and occupation (see Appendix).

Translation into the Greek language kept the principle of EOMEIS-2, which is the use of simple ordinary language to present common situations and was achieved in three stages. a) The 24 items were translated into Greek by the researcher and distribution to four Greek-English language teachers who suggested some corrections. b) the questionnaire was piloted and the language and expressions were discussed with ten Greek Cypriot students from the pilot sample. Minor corrections were made; 
c) finally, the questionnaire was given to two Greek linguists for a final checking and again minor but critical corrections were made.

The scoring rules of the original measure were applied (Adams, 1999) to make comparison with the original study possible. Items were scored by weighting the "strongly agree" response with a value of six and the "strongly disagree" with a value of one. Identity status sub-scales were derived by totalling all six items across the three content domains into a summated sub-scale score of Diffusion, Foreclosure, Moratorium and Achievement. The raw sub-scale scores ranged from a possible low of six to a possible high of thirty-six. Then, the instruments classification rules were applied. According to these rules, participants are assigned to a particular status if their scores on the corresponding status scale exceeds the critical value, which is defined as one standard deviation above the mean. Using a similar strategy, critical values were derived from the data of the present study and were used to classify participants into the four statuses of each identity domain.

Further, an elaborate set of status classification rules allows the collapsing of Transition status (in those cases where the critical value is exceeded for two or more status scales) with "true" statuses. In this way, many participants are eventually assigned to one of Marcia's (1966) four identity four statuses. Participants with scores falling less than one standard deviation above the mean on all four measures are scored as the Low Profile Moratorium. As these adolescents seem to waver between different options, they resemble the Moratorium status. Adams (1999) suggested that "pure" Moratorium and Low-Profile Moratorium should be treated as a single Moratorium status.

The psychometric properties of the part of the EOMEIS-2 are generally good. Reliability, as internal consistency, was measured using Cronbach's alpha. The 
internal consistency for each identity status of the part of EOMEIS-2 version was 0.46 to 0.66 . These alpha scores are lower than the estimations of internal consistency for ideological domain of EOMEIS-2 (0.62 to 0.75$)$ from the basic study of Bennion and Adams (1986) probably because only three domains were used. The estimation of the median Cronbach's alpha from 20 studies ranged from 0.3 to 0.9 and the median was 0.66 (Adams, 1999).

Construct validity of EOMEIS-2 estimated by factor analysis, conducted by Bennion and Adams (1986) and Grotevant and Adams (1984) showed three different factors: one for Achievement, one for Foreclosure and one for Diffusion and Moratorium statuses, which were found to share some variance. Ideally four separate factors, one representing each identity status, would be expected. In this study, the factor analysis was conducted by using Maximum Likelihood extraction and Varimax rotation. For global identity, it gave a blurred picture with only Foreclosure status emerging as a distinct factor, while Moratorium shared some variance with both Achievement and Diffusion. When factor analyses were conducted separately for political, religious and occupational identity the clearest picture emerging from the data, explaining much of the variation was the expected four factors in the domains of occupation and politics (Table 1). For religion, only three factors emerged from the data, Moratorium, Foreclosure and Diffusion.

\section{Insert Table 1}

\section{Results}


Following the scoring procedures of EOMEIS-2, participants were classified into the global identity statuses aggregating their scores across the three identity domains of politics, religion and occupation. Then, applying the same procedures, participants were classified into separate identity statuses for each identity domain. In common with most studies employing EOMEIS-2, less than $50 \%$ of the participants fell into one of the "pure" statuses, (Achievement, Foreclosure, Moratorium and Diffusion). The other 50\% were allocated to a Low-Profile status (participants who do not clearly belong in any one of the "pure" statuses) or a Transition status (participants who belong to more than one "pure" status). Moratorium status had the highest percentage of participants as a result of the inclusion of Low-Profile participants. However, the variability in the classification of participants across global identity and the specific identity domains of politics, religion and occupation is apparent (see Figure 1).

\section{Insert Figure 1}

A goodness of fit test to pair comparisons of the frequencies was conducted to examine whether the distribution across statuses is the same for each specific identity domain and global identity. Three Null Hypotheses were tested and rejected. The distribution across statuses for global identity was significantly different from the distribution of statuses for politics $\left(\chi^{2}=22.09, d f=3, p<.01\right)$, religion $(\chi 2=12.27, d f=3$, $p<.01)$ and occupation $\left(\chi^{2}=34.44, d f=3, p<.01\right)$.

\section{Comparison of Greek Cypriot and North American adolescents}

There were significant differences in identity status comparing Greek Cypriot adolescents and American adolescents of the same age from Skorikou and 
Vondracek's (1998) study on political, religious and occupational identities (Table 2). In political identity, the great majority of young Greek Cypriots were politically engaged (i.e., they were significantly more likely to belong to Achievement, Moratorium and Foreclosure statuses) than their American counterparts who showed indifference to politics (they were more likely to be in Diffusion status). In religious identity, young Greek Cypriots were more likely to belong to Achievement and Moratorium statuses; where their American counterparts were more likely to belong to Foreclosure and Diffusion statuses. In occupational identity the only difference was in Diffusion status, more common amongst American young people.

Insert Table 2

Associations between global and domain identities and adolescents' type of school

The relationship between meso-contextual variables (type of school and SES) and identity was examined, both at the global and domain specific level. Associations between different types of schools and global, religious and occupational identities were significant as shown in Table 3. Students form state technical schools were more likely to belong to Diffusion status in global, religion and occupational identity. In religious identity students at state schools were particularly likely to be achieved. In occupational identity students from private schools were more likely to be in Moratorium status. Interestingly, the association between participants' type of school and political identity is not statistically significant $(\chi 2=10.27, d f=6)$.

Insert Table 3 
Results showed that there were significant associations between SocioEconomic Status and religious and occupational identities that were not apparent when examining global identity (see Table 4). Moreover, the relationship between Socio-Economic Status and religious and occupational identity differed. In religious identity, students from upper SES were more likely to be diffused. In occupational identity working class students were more likely to be diffused and upper class students in Moratorium.

Insert Table 4

Associations between global and domain identities and adolescents' gender

The relationship between micro-contextual variables (gender) and identity was examined, both at the global and domain specific level. The association between adolescents' gender and global, political, religious and occupational identities were significant as shown in Table 5. However, the direction of the difference diverged and the separate investigation of gender differences in each identity domain revealed a more complex picture than provided by global identity on its own. There was an association between gender and global identity in two of the four statuses. Young women were more likely to belong to Moratorium status and young men were more likely to belong to Diffusion status. However, this picture was not reflected in all the three identity domains from which global identity was aggregated. In political identity the differences between gender and identity status was unrelated to the results of global identity. Young men were more likely to be foreclosed in their political identity, whereas females were more likely to be diffused. Only the associations of religious identity with gender showed the same significant differences between males and females as global identity. Finally, in occupational identity young women were 
more likely to belong to Moratorium status and young men were more likely to belong to Diffusion status, which reflects the associations of global identity. However, females were also more likely to belong to Achievement status.

\section{Insert Table 5}

\section{Discussion}

Overall results and tests from part of EOMEIS-2 questionnaire, conducted in an unresearched culture (Greek Cypriot society) replicated results that global identity is not synonymous with domain specific identities. Thus, our study supports the recommendations of De Haan \& Schulenberg (1997) and Goossens (2001) for the separate study of each identity domain and is consistent with self-concept theory, which has a long tradition of considering the specific self-concepts as distinguishable entities (Harter, 1996). Moreover the study improves on previous efforts by showing that the identity domains of politics, religion and occupation in relation to gender, type of school and SES provide a more complex and heterogeneous picture of identity than can be observed by looking at global identity alone. Therefore, in studying identity in context, domain specific identities reveal the subtleties of macro-, mesoand micro- contextual influences.

More importantly the present study facilitates understanding of the way to macro-context relates to identity status and offers an insight into identity formation in a cultural context which differs from the "dominant" Western one. The hypothesis that Greek Cypriot adolescents would be more "advanced" in their political and religious identities than the same age American adolescents (Skorikou \& Vondracek, 1998) was confirmed. Obviously, macro-contextual factors, namely the tense political 
situation, the value placed on political participation and the strong Christian Orthodox tradition are encouraging young Greek Cypriots to explore and to form commitments in these specific domains of identity. Their American and European counterparts are more likely to be in Foreclosure (Jackson et al., 1990; Meilman, 1979; Skorikou \& Vondracek, 1998) or in Diffusion statuses (Goossens, 2001; Skorikou \& Vondracek, 1998) in their religious identity and in Diffusion status in their political identities (Archer, 1982; Goossens, 2001; Jackson et al., 1990; Meilman, 1979; Skorikou \& Vondracek, 1998). Baumeister and Muraver (1996) proposed that adolescents in Western societies have greater responsibility for creating their own identity following the erosion of tradition and religion in contemporary Western societies. This study shows that the presence of strong value bases in society facilitates young people in forming their identities. Cote and Levine's (2002) division of identity statuses according to different types of society cannot be applied to some contemporary societies (e.g., Greek Cypriot society) that combine modernization with tradition and where Achievement and Moratorium statuses emerge as individuals are encouraged to make their own decisions but within the structures of existing institutions.

In occupational identity American and Greek Cypriot adolescents are more similar, consistent with other studies (Bosma, 1992; Bynner, 1998; Kroger, 1993; Schulenberg, Goldstein, \& Vondracek, 1991; Silbereisen, Vondracek, \& Berg, 1997), which conclude that the formation of occupational identity is a priority in adolescence.

The examination of the impact of the three different types of secondary education in Cyprus on identity statuses in the domains of religion and occupation showed interesting differences and shed light in this new research area. Religious identity seems to be influenced by the religious education (RE) offered in each type of 
school. State school students were more likely to be in Achievement status and less likely to be in Diffusion status. State schools in Cyprus provide more hours of religious education, where a discussion of religious issues, as well as the position of the Christian Orthodox church is encouraged. According to Benson et al (1989), 1,000 hours of classroom instruction in religion had a long term impact on adolescent religiousness. State technical and private schools provide religious education to a lesser degree, consistent with the findings that state technical and private school students were more likely to be in Diffusion status.

Concerning occupational identity, the hypothesis that state technical school student would be more "advanced" was not confirmed. On the contrary, state technical school students were more likely to be in Diffusion status and less likely to be in Moratorium status. This finding was surprising, as their vocational orientation was expected to clarify their future occupational identities, so this issue needs further investigation. Probably this investigation should concern occupational identity content in terms of work values, such as materialism, self-actualisation and socialresponsibility (Ros, Schwartz, \& Surkiss, 1999). The evidence that private school students were more likely to be in Moratorium status and less likely to be in Achievement status, seems to parallel their upper SES. Students from private schools typically come from the more affluent end of society, where career and employment options are much wider and the school ethos encourages academic confidence amongst students. Bynner (1998) suggested that children from affluent homes who acquire both the cognitive and non-cognitive elements of identity capital, are more likely to be assured of future employment.

This study contributes to an under-investigated area, namely the impact of Socio-Economic Status on political, religious and occupational identities. Upper class 
and working class participants were opposed. In religious identity, upper class participants were more likely to be in Diffusion status whereas working class participants were more likely to be in Foreclosure status. In occupational identity, upper class participants were more likely to be in Moratorium status and less likely to be in Diffusion status, whereas working class participants were the opposite. In an empirical test of identity capital, Cote (1997) was able to show that those from the upper social class had the affluence to afford a longer Moratorium period. Working class participants were more likely to be diffused. This may be the explanation for why students at state technical school, predominantly from working class backgrounds, were diffused in their occupational identity. Unfortunately, due to the sample size restriction it was not possible to test this in the present study (i.e., the sample size for state technical school participants was small due to the proportionate sampling). Yoder (2000) proposed that Socio-Economic Status, educational opportunities and gender limit the individual's exploration or/and commitment in all or some domains. Social class showed less discriminatory power than the combination of education and gender variables, probably because Cypriot social class structure has a short history and parents invest in education to ensure the social mobility of their children.

Political identity status had no significant relationship with type of school or SES showing that the intense political climate structured young Greek Cypriots to be politically engaged, independent of their social and cultural capital. An opposite hypothesis that needs investigation might be that EOMEIS-2 is not sensitive enough to capture these meso-level differences, while another investigation measuring political identity content in terms of political values would identify difference related to type of school and SES. 
Finally, the examination of gender showed striking differences, which reflect socio-contextual expectations and restrictions. Both hypotheses on gender were confirmed. Specifically, the Greek Cypriot males in this study were more likely to be foreclosed in their political identity. Young men, conscripted for over 2 years to the armed forces, are expected to defend Greek Cypriot society in a most tangible way, encouraging political commitment but not political exploration. Young Greek Cypriot women were more likely to be in a more "advanced" status (e.g., Moratorium), in accordance with Donelson's (1999) review of religious identity, which finds females to be generally somewhat more religious than males. Context reinforces religiosity by expecting women to be concerned with keeping the religious customs; high exploration in religion signifies interest. It might be anticipated that strict cultural expectations would lead to Foreclosure but it would appear that this was not the perception of these young women. Perhaps this marks the response of modernisation of Cyprus society to Orthodoxy. In occupational identity males were more likely to belong to Diffusion status and females were more likely to belong to Achievement status. This reflects the specific contextual situation, where males, after secondary school, have to serve in the army postponing any occupational plans, whereas females proceed to higher education or a job.

The present study exhibits some weaknesses concerning the research methods used that need to be addressed. Firstly, EOMEIS-2 is self-report, giving to participants ready made options and restrict their respond only to these options. Secondly, less than $50 \%$ of young Greek Cypriots were classified in one of the four pure identity statuses; over $30 \%$ were classified as Low Profile and over $20 \%$ were classified as Transitional in their political, religious and occupational identities. This is a known feature in EOMEIS studies (Adams, 1999) that shows both a methodological 
weakness and the applicability of the four identity categories to all participants. There is also a problem with whether some items of the instrument measure the assigned identity statuses within the specific culture. Especially, young Greek Cypriots interpreted the items relating to religion as referring only to Christian Orthodoxy and this interpretation affected mostly the reliability of the two religion Achievement items (see Appendix). Thirdly, the Cronbach's alpha for Diffusion is low (.46) indicates that the scale probably measures more than a single construct. So caution should be put in the interpretation of Diffusion status results. Finally, the crosssectional design of the study is also a weakness, therefore a longitudinal design, one that could follow participants in early adulthood and beyond would offer more complete knowledge on how micro, meso and macro contexts influence identity across the lifespan.

In conclusion, the present study suggests the value of studying identity at the level of domain rather than as a global entity and begins to reveal the way that micro, meso and macro contexts shape adolescents identities.

\section{Appendix}

EOMEIS-2 items covering the domains of politics, religion and occupation

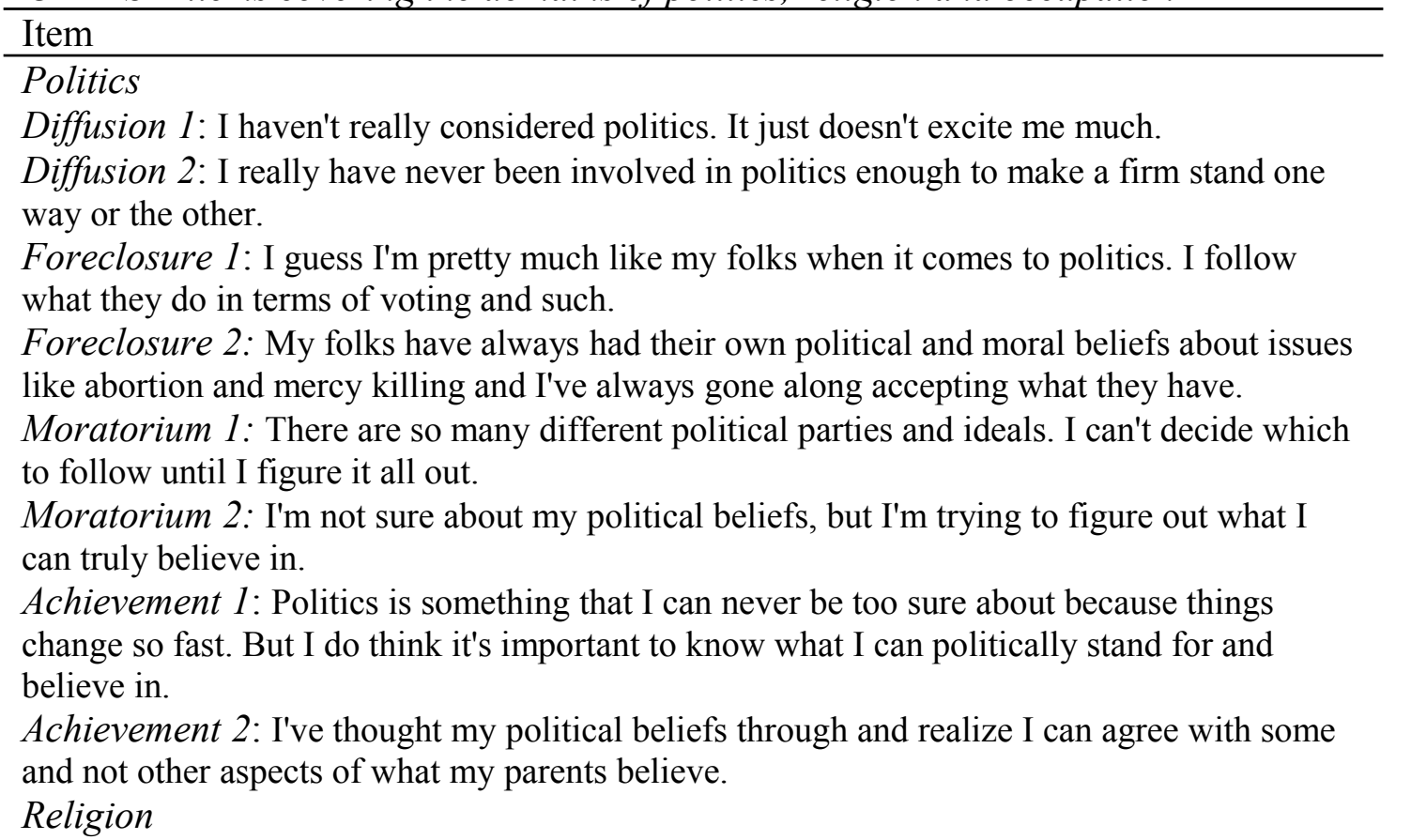


Diffusion 1: When it comes to religion I just haven't found anything that appeals and I don't really feel the need to look.

Diffusion 2: I don't give religion much thought and it doesn't bother me one-way or the other.

Foreclosure 1: I attend the same church as my family has always attended. I've never really questioned why.

Foreclosure 2: I've never really questioned my religion. If it's right for my parents it must be right for me.

Moratorium 1: I'm not sure what religion means to me. I'd like to make up my mind but I'm not done looking yet.

Moratorium 2: Religion is confusing to me right now. I keep changing my views on what is right and wrong for me.

Achievement 1: A person's faith is unique to each individual. I've considered and reconsidered it myself and know what I can believe.

Achievement 2: I've gone through a period of serious questions about faith and can now say I understand what I believe in as an individual.

Occupation

Diffusion 1: I haven't chosen the occupation I really want to get into, and I'm just working at what is available until something better comes along.

Diffusion 2: I'm not really interested in finding the right job, any job will do. I just seem to flow with what is available.

Foreclosure 1: I might have thought about a lot of different jobs, but there's never really been any question since my parents said what they wanted.

Foreclosure 2: My parents decided a long time ago what I should go into for employment and I'm following through their plans.

Moratorium 1: I'm still trying to decide how capable I am as a person and what work will be right for me.

Moratorium 2: I just can't decide what to do for an occupation. There are so many possibilities.

Achievement 1: It took me a while to figure it out, but now I really know what I want for a career.

Achievement 2: It took me a long time to decide but now I know for sure what direction to move in for a career.

\section{References}

